\title{
The early change of SOFA score as a prognostic marker of 28-day sepsis mortality: analysis through a derivation and a validation cohort
}

\author{
Eleni Karakike' ${ }^{1}$ Evdoxia Kyriazopoulou' ${ }^{1}$ Iraklis Tsangaris ${ }^{2}$, Christina Routsi ${ }^{3}$, Jean-Louis Vincent ${ }^{4}$ and \\ Evangelos J. Giamarellos-Bourboulis ${ }^{1 *}$ (D)
}

\begin{abstract}
Background: Since the Sepsis-3 criteria, change in Sequential Organ Failure Assessment (SOFA) score has become a key component of sepsis identification. Thus, it could be argued that reversal of this change $\left(\Delta_{\text {SOFA }}\right)$ may reflect sepsis response and could be used as measure of efficacy in interventional trials. We aimed to assess the predictive performance of $\Delta_{\text {SOFA }}$ for 28-day mortality.

Methods: Data from two previously published randomized controlled trials were studied: the first reporting on patients with severe Gram-negative infections as a derivation cohort and the second reporting on patients with ventilator-associated pneumonia as a validation cohort. Only patients with sepsis according to the Sepsis-3 definition were included in this analysis. SOFA scores were calculated on days 1, 2, 3, 5, 7, 14, and 28.

Results: We included 448 patients within the derivation cohort and 199 within the validation cohort. Mean SOFA scores on day 1 were $6.06 \pm 4.07$ and $7.84 \pm 3.39$, and 28 day mortality $22.8 \%$ and $29.6 \%$, respectively. In the derivation cohort, the earliest time point where $\Delta_{\text {SOFA }}$ score predicted mortality was day 7 (AUROC (95\% Cl) 0.84 $(0.80-0.89) ; p<0.001)$. The best tradeoff for prediction was found with $25 \%$ changes ( $78 \%$ sensitivity, $80 \%$ specificity); less than $25 \%$ decrease of admission SOFA was associated with increased mortality (odds ratio for death 14.87). This finding was confirmed in the validation cohort.
\end{abstract}

Conclusions: $\Delta_{\text {SOFA }}$ on day 7 is a useful early prognostic marker of 28-day mortality and could serve as an endpoint in future sepsis trials alongside mortality.

Trial registration: ClinicalTrials.gov numbers NCT01223690 and NCT00297674

Keywords: Sequential Organ Failure Assessment (SOFA), Delta change, Sepsis, Mortality, Trial endpoints

\section{Background}

In the light of numerous inconclusive interventional clinical trials in sepsis during the past two decades, the framework of those trials is to be revised [1-4]. Allcause mortality after 28 days has traditionally been the primary endpoint in these trials. However, with recent improvements in standard-of-care therapy, 28-day mortality is strongly dependent from other variables

\footnotetext{
* Correspondence: egiamarel@med.uoa.gr

${ }^{1} 4$ th Department of Internal Medicine, Attikon University Hospital, National and Kapodistrian University of Athens, 1 Rimini Street, 12462 Athens, Greece Full list of author information is available at the end of the article
}

such as comorbid conditions and the adverse events of multiple interventions [5]. As such, it is reasonable that alternative endpoints need to be developed for sepsis. These endpoints need to provide earlier and accurate evaluation of the treatment effect under study.

Since sepsis is triggered by an infection, the endpoint of sepsis trials may be influenced by the attitude of regulatory bodies to focus new registration trials of antimicrobial agents towards early efficacy. The main example towards this end is the joint initiative between the Food and Drug Administration (FDA) with the Biomarkers Consortium of the Foundation for the National

(C) The Author(s). 2019 Open Access This article is distributed under the terms of the Creative Commons Attribution 4.0 International License (http://creativecommons.org/licenses/by/4.0/), which permits unrestricted use, distribution, and 
Institutes of Health (FNIH) on the update of primary endpoint definitions for non-inferiority trials for the management of infectious diseases. More precisely, the former test-of-cure visit usually taking place 7-14 days after end of treatment was replaced by the early response 48-72 h after start of treatment for acute bacterial skin and soft structure infections [6] and 3-5 days after start of treatment for community-acquired pneumonia [7], while efforts are being made to expand this concept to hospital-acquired and ventilator-associated pneumonia [8, 9]. However, in order to develop a similar early endpoint for sepsis, it is mandatory that this endpoint is a predictor of 28-day mortality, i.e., the salient sequelum of sepsis and eventually of 90-day mortality that has recently emerged as a relevant clinical endpoint [10]. With the Sepsis-3 classification criteria, the Sequential Organ Failure Assessment (SOFA) score is used as a measure of sepsis-associated organ dysfunction. As a consequence, it is reasonable to define the earliest time point during the course of the disease where a clinical meaningful change of the baseline SOFA score is achieved.

The present study tries to define (a) the earliest time point during the course of sepsis where SOFA score changes can predict 28-mortality and (b) the cutoff change of baseline SOFA score that may be considered an early sign of sepsis resolution. The association of SOFA score changes with 90-day mortality is also assessed. In order to achieve so, we used two independent prospective cohorts of patients: the first as a derivation cohort and the second as a validation cohort.

\section{Patients and methods}

\section{Study populations}

We retrospectively analyzed clinical data from a cohort of patients with sepsis, according to the 1991 sepsis definitions (derivation cohort) [11]; a second independent cohort using the 1991 sepsis definitions served as validation dataset for the primary hypothesis. Both cohorts were part of previously published multicenter randomized controlled trials comparing clarithromycin to placebo as adjunctive immunomodulatory treatment in sepsis $[12,13]$.

The derivation cohort included patients with Gramnegative sepsis, enrolled in a prospective double-blind, placebo-controlled randomized clinical trial (RCT) studying the efficacy of intravenous clarithromycin in 28-day mortality. Patients were recruited from July 2007 to August 2011 in six departments (two intensive care units-ICUs, three medical wards, and one surgical ward) in five tertiary teaching hospitals in Greece. Patients were suffering from acute pyelonephritis or intra-abdominal infections or primary Gram-negative bacteremia [12] (ClinicalTrials.gov NCT01223690). Since the 28-day mortality of patients allocated to the placebo arm and of patients allocated to the clarithromycin arm did not differ, both arms were analyzed together for the purpose of this study.

The validation cohort consisted of patients with ventilator-associated pneumonia (VAP), enrolled in an RCT in two ICUs (one patient enrolled in one medical ward has not been included in the present study) in two tertiary teaching hospitals in Greece, from June 2004 to November 2005 (ClinicalTrials.gov NCT00297674) [13]. Since the 28-day mortality of patients allocated to the placebo arm and of patients allocated to the clarithromycin arm did not differ, both arms were analyzed together.

All medical and nursing charts of the derivation cohort were retrospectively reviewed, and components of SOFA score for each system (respiratory, coagulation, liver, cardiovascular, central nervous, and renal) were collected. Serial SOFA scores were calculated initially on day 1 (initial SOFA) and on days 2, 3, 5, 7, 14, and 28 after enrollment in the study.

For the purposes of this study, patients of each cohort who were meeting the Sepsis-3 criteria were identified; only those participated in this analysis. For the calculation of serial SOFA scores, when the Glasgow Come Scale (GCS) was not evaluable due to sedation for mechanical ventilation, the GCS immediately before mechanical ventilation was used. Patients discharged from hospital or deceased before day 28 were censored to the last known SOFA score. Delta SOFA $\left(\Delta_{\text {SOFA }}\right)$ for any follow-up day was provided by the formula: (SOFA score of the follow-up day-initial SOFA score) $\times 100 /$ day 1 SOFA, and it was expressed as percentage.

The outcome measure in both cohorts was the earliest time point where the change of SOFA score was associated with 28-day mortality. The association of this change with 90-day mortality was a secondary endpoint.

\section{Statistical analysis}

Categorical values were presented as percentages, and continuous variables with normal distribution as mean and standard deviation $( \pm \mathrm{SD})$. Categorical variables were compared using the two-sided Fisher exact test, whereas quantitative variables were assessed using Student's $t$ test or the non-parametric Mann-Whitney test, as appropriate. The predictive capacity of different follow-up day $\Delta_{\text {SOFA }}$ for mortality was evaluated with the area under the respective receiver operator characteristics (AUROC) curves and 95\% confidence intervals (CIs). The optimal cutoff value for prediction of 28-day mortality was calculated using Youden's index. The $\Delta_{\text {SOFA }}$ was expressed by medians and 95\% CIs; comparisons between survivors and non-survivors were done by the Mann-Whitney $U$ test. Breslow-Day's test was used to compare the 
performance of this cutoff value between the derivation and validation cohorts. A $p$ value lower than 0.05 was considered statistically significant. All $p$ values were twosided. Statistical analyses were performed using SPSS version 25.0 software.

\section{Results}

The study flow charts for both cohorts are shown in Fig. 1. A total of 448 of patients of the derivation cohort and 199 patients of the validation cohort could be classified as sepsis according to the Sepsis-3 criteria and were included in the analysis. Demographic baseline data of the two cohorts differed significantly (Table 1 ).

\section{Primary endpoint}

The ROC curves of the performance of $\Delta_{\text {SOFA }}$ of followup days for the prediction of 28-day mortality in the derivation cohort are shown in Fig. 2a. When the AUROCs of $\Delta_{\text {SOFA }}$ of follow-up days were compared, it was found that the earliest time point when the achieved AUROC was greater than previous days was on day 7 (Fig. 2b). When the absolute $\Delta_{\text {SOFA }}$ scores were compared over time between survivors and non-survivors, despite the significantly greater decreases in survivors from nonsurvivors found by non-parametric statistics at all time points, a great overlap of values was shown (Fig. 2c). This led us to consider the percentage change of baseline SOFA as a more appropriate expression of the sepsis course than the absolute $\Delta_{\text {SOFA }}$. To this end, our analysis focused on the development of a specific value of $\Delta_{\text {SOFA }}$ of day 7 as an early predictor of 28-day mortality. The analysis using the Youden index showed that a 25\% cutoff value could discriminate non-survivors from survivors with sensitivity $78.4 \% \quad(95 \%$ CI $\quad 69.0-85.7 \%$ ), specificity $80.3 \%$ (95\% CI $75.7-84.3 \%$ ), positive predictive value $54.1 \%$ (95\% CI $45.7-62.2 \%$ ), and negative predictive value $92.7 \%$ (95\% CI 89.0-95.2\%).

Overall, in the derivation cohort, 148 (33\%) patients had less than $25 \%$ decrease of SOFA score on day 7 and
$300(77 \%)$ patients had at least $25 \%$ decrease of initial SOFA score on day 7. Mortality after 28 days was $54.1 \%$ and $7.3 \%$, respectively $\left(p=1.8361 \times 10^{-27}\right)$. The OR for death after 28 days with a decrease of initial SOFA on day 7 less than 25\% was 14.87 (95\% CI 8.65-25.54). Similarly, the OR for death in the validation cohort was 6.95 (95\% CI 2.05-23.55) ( $p$ value of the Breslow-Day test of homogeneity 0.250 ) (Table 2).

\section{Secondary endpoint}

After ROC analysis, the day $7 \Delta_{\text {SOFA }}$ in the derivation cohort yielded an AUROC of $0.847(0.807-0.886 ; p=$ $5.11 \times 10^{-29}$ ) for predicting 90-day mortality. When applying the cutoff of less than $25 \%$ decrease, this was associated with an OR of 13.20 for death after 90 days $\left(95 \%\right.$ CI $\left.8.01-21.76 ; p=4.78 \times 10^{-28}\right)$. Table 3 describes the performance characteristics of the cutoff in predicting 90-day mortality in both cohorts.

\section{Post hoc analysis}

Although the validation cohort involved 199 with VAP all of whom were under mechanical ventilation, the derivation cohort comprised both mechanically $(n=71)$ and non-mechanically ventilated patients $(n=377)$ on study enrollment. The 28-day mortality among mechanically ventilated patients with at least $25 \%$ decrease of initial SOFA score and among mechanically ventilated patients with less than $25 \%$ decrease of initial SOFA score was $11.5 \%$ and $37.8 \%$, respectively $(p=0.027)$. The respective 28-day mortality among the nonmechanically ventilated patients was $7.0 \%$ and $60.0 \%$, respectively $\left(p=1.1 \times 10^{-26}\right)$.

Due to the significant baseline differences between the derivation and validation cohorts and in order to assess the robustness of the above findings, a post hoc analysis has been performed, by merging both initial cohorts and randomly splitting them into cohort $\mathrm{A}$ and cohort B. It needs to be outlined that patients of both original cohorts were recruited before 2012 (the
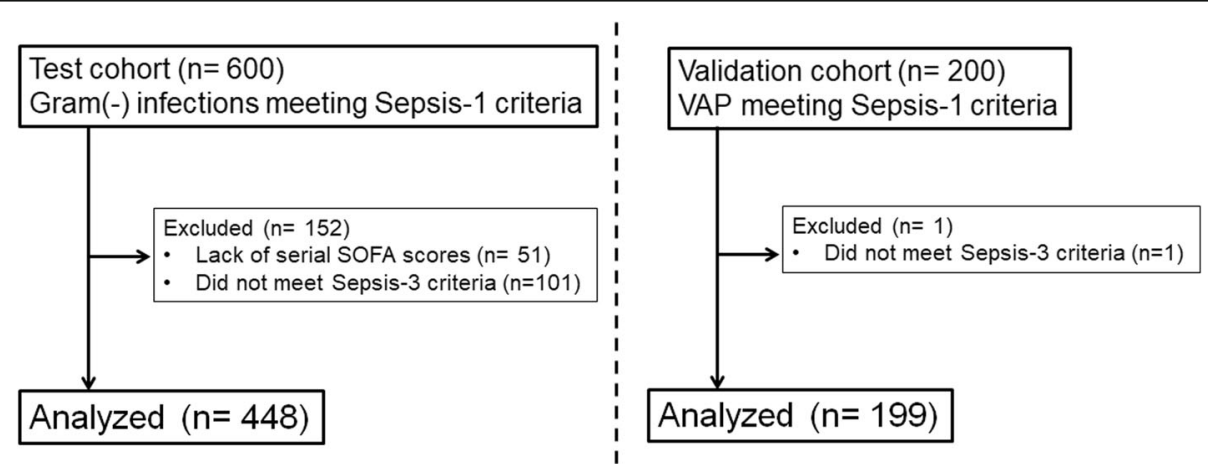

Fig. 1 Flow chart. VAP, ventilator-associated pneumonia; SIRS, systemic inflammatory response syndrome; SOFA, sequential Organ Failure Assessment 
Table 1 Comparative baseline demographics of the two cohorts

\begin{tabular}{|c|c|c|c|}
\hline & Derivation cohort $(n=448)$ & Validation cohort $(n=199)$ & $p$ value \\
\hline Male gender, $n(\%)$ & $213(47.5)$ & $147(73.9)$ & $<0.001$ \\
\hline Age (years, mean $\pm S D$ ) & $71.7 \pm 16.6$ & $58.4 \pm 19.1$ & $<0.001$ \\
\hline SOFA score (mean \pm SD) & $6.1 \pm 4.1$ & $7.8 \pm 3.4$ & $<0.001$ \\
\hline APACHE II score (mean \pm SD) & $15.1 \pm 7.4$ & $17.1 \pm 5.7$ & 0.001 \\
\hline $\mathrm{CCl}($ mean $\pm \mathrm{SD})$ & $4.1 \pm 2.5$ & $2.6 \pm 1.7$ & $<0.001$ \\
\hline $\mathrm{PaO} 2 / \mathrm{FiO} 2$ ratio (mean $\pm \mathrm{SD}$ ) & $298.8 \pm 112.6$ & $218.5 \pm 98.0$ & $<0.001$ \\
\hline Mechanical ventilation, $n(\%)$ & $90(20.1)$ & $199(100)$ & $<0.001$ \\
\hline \multicolumn{4}{|l|}{ Characteristics of MV population } \\
\hline Tidal volume $(\mathrm{ml} / \mathrm{kg}$, mean $\pm \mathrm{SD})$ & $6.6 \pm 0.9$ & $6.5 \pm 0.9$ & 0.179 \\
\hline PEEP level (mmHg, mean \pm SD) & $5.7 \pm 0.9$ & $6 \pm 0.9$ & 0.011 \\
\hline $\mathrm{PaO} 2 / \mathrm{FiO} 2$ ratio $($ mean $\pm \mathrm{SD})$ & $252.7 \pm 113.7$ & $218.5 \pm 98.0$ & 0.020 \\
\hline Duration of MV (days, mean \pm SD) & $14.5 \pm 13.8$ & $14.7 \pm 10.4$ & 0.346 \\
\hline \multicolumn{4}{|l|}{ Underlying infection, $n(\%)$} \\
\hline Acute pyelonephritis & $207(46.2)$ & $0(0)$ & NA \\
\hline Acute intra-abdominal infection & $162(36.2)$ & $0(0.0)$ & NA \\
\hline Primary Gram-negative bacteremia & $71(15.8)$ & $0(0.0)$ & NA \\
\hline $\begin{array}{l}\text { Secondary Gram-negative bacteremia } \\
\text { (other than urinary or intra-abdominal) }\end{array}$ & $8(1.8)$ & $0(0.0)$ & 0.107 \\
\hline Ventilator-associated pneumonia & $0(0)$ & $199(100.0)$ & NA \\
\hline Early (<7 days of MV) & & $84(42.2)$ & \\
\hline Late (> 7 days of MV) & & $115(57.8)$ & \\
\hline Septic shock, $n(\%)$ & $88(19.6)$ & $85(42.7)$ & $<0.001$ \\
\hline ARDS, $n(\%)$ & $136(30.4)$ & $150(75.4)$ & $<0.001$ \\
\hline ICU admission, $n$ (\%) & $90(20.1)$ & $198(99.5)$ & $<0.001$ \\
\hline ICU LOS (days, mean \pm SD) & $45.3 \pm 94.3$ & $36.9 \pm 34.4$ & 0.317 \\
\hline Hospital LOS (days, mean \pm SD) & $20.0 \pm 47.6$ & $51.7 \pm 47.5$ & $<0.001$ \\
\hline For ICU-admitted population & $49.6 \pm 98.9$ & $51.7 \pm 47.5$ & 0.006 \\
\hline For non-ICU-admitted population & $12.61 \pm 0.9$ & NA & \\
\hline ICU mortality, n (\%) & $49(54.4)$ & $89(44.9)$ & 0.162 \\
\hline Hospital mortality, n (\%) & $123(27.5)$ & $110(55.3)$ & $<0.001$ \\
\hline 28-day mortality, n (\%) & $102(22.8)$ & $59(29.6)$ & 0.075 \\
\hline 90-day mortality, $n$ (\%) & $118(26.3)$ & $153(76.9)$ & $<0.001$ \\
\hline
\end{tabular}

Abbreviations: ARDS: Acute Respiratory Distress Syndrome, SD standard deviation, SOFA Sequential Organ Failure Assessment, APACHE Acute Physiology and Chronic Health Evaluation, CCI Charlson's comorbidity index, ICU: Intensive Care Unit, LOS: Length of Stay, MV: mechanical ventilation, NA not applicable

Table 2 Comparative prognostic performance for 28-day mortality of the less than $25 \%$ SOFA decrease cutoff on day $7 \Delta_{\text {SOFA }}$ between the derivation and the validation cohorts

\begin{tabular}{llll}
\hline & Derivation cohort $(95 \% \mathrm{Cl})$ & Validation cohort $(95 \% \mathrm{Cl})$ & $p$ value \\
\hline Sensitivity & $78.4 \%(69.0-85.7)$ & $93.2 \%(84.7-98.7)$ & 0.06 \\
Specificity & $80.3 \%(75.7-84.3)$ & $37.9 \%(20.1-35.4)$ & $2.87 \times 10^{-28}$ \\
PPV & $54.1 \%(45.7-62.2)$ & $38.7 \%(27.7-43.1)$ & 0.01 \\
NPV & $92.7 \%(89.0-95.2)$ & $93.0 \%(79.0-98.1)$ & 1.00 \\
\hline
\end{tabular}

Abbreviations: $C l$ confidence interval, $N P V$ negative predictive value, $P P V$ positive predictive value 

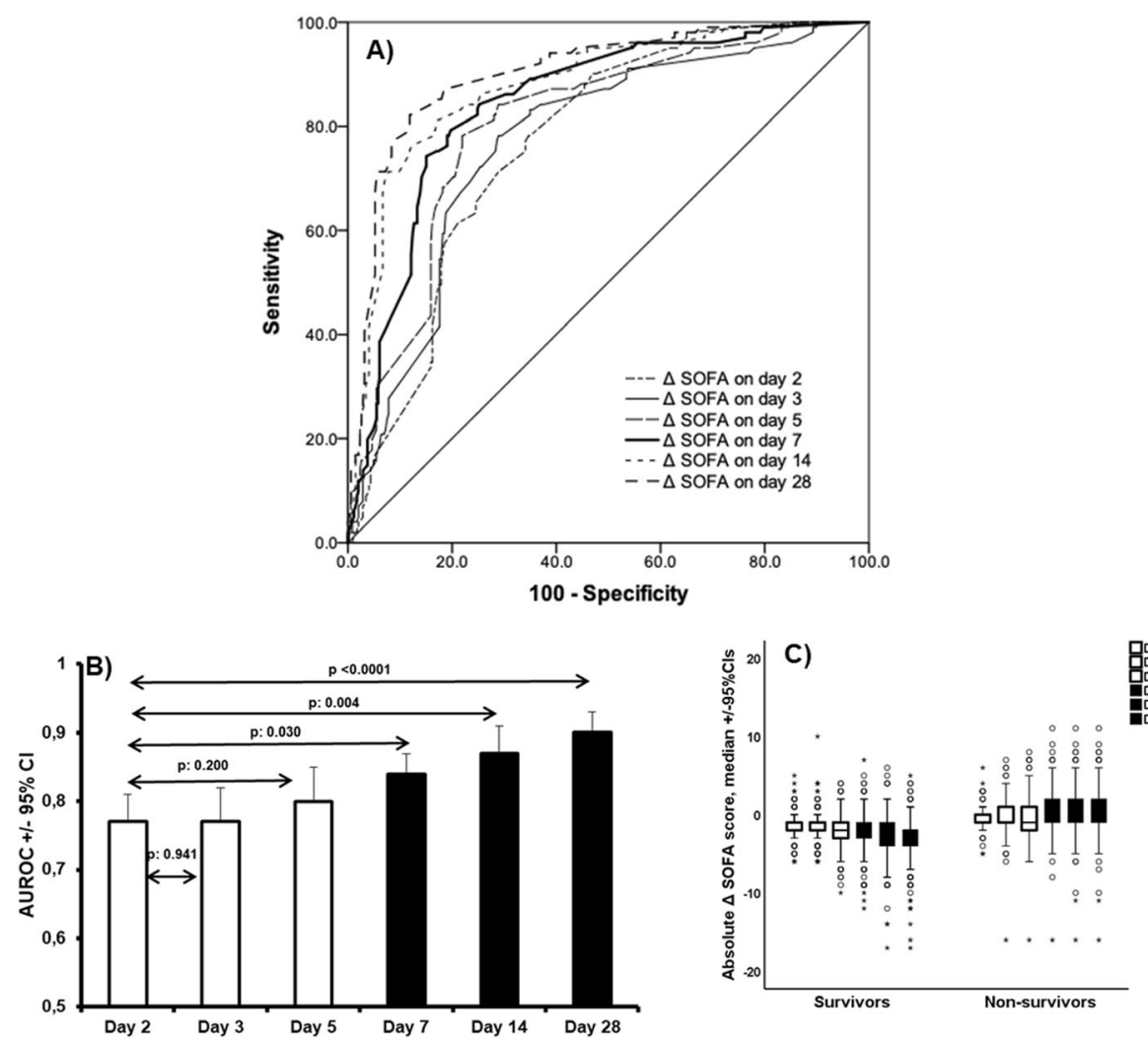

Fig. $2 \Delta_{\text {SOFA }}$ on follow-up days as predictor of 28-day mortality in the derivation cohort. a Receiver operating characteristic (ROC) curves for the association of change from initial SOFA ( $\Delta_{\text {SOFA }}$ ) with 28-day mortality. $\mathbf{b}$ Comparisons of AUROCS of $\Delta_{\text {SOFA }}$ of follow-up days to $\Delta_{\text {SOFA }}$ of day 2 . $p$ values of the indicated comparisons are provided. c Median $\Delta_{\text {SOFA }}$ scores on follow-up days in survivors and non-survivors. Statistically significant differences at the level of $p<0.0001$ were found between survivors and non-survivors at all studied time points. AUROC, area under the ROC; Cl, confidence interval

first in the years 2004-2005 and the second in the years 2007-2011). The standard-of-care for patients remained approximately the same between these two periods since the Surviving Sepsis Campaign guidelines remained largely unchanged between 2004 and 2008 as also where national recommendations for antimicrobial use. Baseline characteristics of the new cohorts shown in Additional file 1: Table S1 did not differ. The $25 \%$ change of initial SOFA score worked equally well for the prediction of both 28-day and 90-day mortality in both cohorts A and B (Table 4 and Additional file 2: Table S2, respectively).
Another concern was that some investigators handle SOFA score for deceased patients as the last observation carried forward, while others set the score to 24 in case of death. Using the second approach in the derivation cohort, it was found that 28-day mortality among 295 patients with at least $25 \%$ decrease of initial SOFA score was $6.1 \%$; this was $56.2 \%$ among 153 patients with less than $25 \%$ decrease of the initial SOFA score.

\section{Discussion}

To the best of our knowledge, this is the first study to report a specific cutoff of $25 \%$ decrease of SOFA score

Table 3 Comparative prognostic performance for 90-day mortality of the less than 25\% SOFA decrease cutoff on day $7 \Delta_{\text {sofa }}$ between the derivation and validation cohorts

\begin{tabular}{llll}
\hline & Derivation cohort $(95 \% \mathrm{Cl})$ & Validation cohort $(95 \% \mathrm{Cl})$ & $p$ value \\
\hline Sensitivity & $74.6 \%(69.5-83.4)$ & $77.1 \%(69.5-83.4)$ & 0.63 \\
Specificity & $81.8 \%(77.1-85.7)$ & $47.8 \%(33.1-62.9)$ & $2.00 \times 10^{-5}$ \\
PPV & $59.5 \%(51.1-67.4)$ & $83.1 \%(75.7-88.7)$ & $9.00 \times 10^{-5}$ \\
NPV & $90.0 \%(85.9-93.1)$ & $38.6 \%(26.3-52.4)$ & $1.54 \times 10^{-27}$ \\
\hline
\end{tabular}

Abbreviations: $C l$ confidence interval, $N P V$ negative predictive value, $P P V$ positive predictive value 
Table 4 Prognostic performance for 28-day mortality of the 25\% SOFA decrease cutoff on day $7 \Delta_{\text {SOFA }}$ using post-hoc derivation and validation cohorts

\begin{tabular}{|c|c|c|c|c|c|c|}
\hline & \multicolumn{2}{|l|}{ Cohort A } & \multirow[t]{2}{*}{ Total } & \multicolumn{2}{|l|}{ Cohort B } & \multirow[t]{2}{*}{ Tota } \\
\hline & Non-survivors (n) & Survivors $(n)$ & & Non-survivors $(n)$ & Survivors $(n)$ & \\
\hline$\geq 25 \%$ SOFA decrease & $\begin{array}{l}74 \\
\text { Sens: } 86.0 \% \\
\text { PPV: } 51.7 \%\end{array}$ & 69 & 143 & $\begin{array}{l}61 \\
\text { Sens: } 91.3 \% \\
\text { PPV: } 41.5 \%\end{array}$ & 86 & 147 \\
\hline \multirow[t]{2}{*}{$<25 \%$ SOFA decrease } & 12 & $\begin{array}{l}169 \\
\text { Spec:71.0\% } \\
\text { NPV: } 93.4 \%\end{array}$ & 181 & 14 & $\begin{array}{l}162 \\
\text { Spec: } 65.3 \% \\
\text { NPV: } 92.0 \%\end{array}$ & 176 \\
\hline & 86 & 238 & 324 & 75 & 248 & 323 \\
\hline
\end{tabular}

Abbreviations: NPV negative predictive value, PPV positive predictive value, Sens sensitivity, Spec specificity

as the earliest significant surrogate of 28-day mortality using a derivation and a validation cohort. The cutoff remained robust in all subsequent analyses and subgroup evaluations, despite the fact that the used cohorts differed considerably in baseline characteristics, indicating that the elaborated endpoint may be generalizable.

Previous studies have shown that serial SOFA measurements are predictors of mortality on both days 3 and 5 of follow-up [14, 15]. A cohort study of 20,007 critically ill patients in Canada reported that the slope of the SOFA score between days 1 and 7 was higher and better associated with final outcome (both ICU and hospital mortality) than was the average rate of change at later time points (between days 8 and 14) [16]. According to the authors, any increase between days 1 and 5 (defined as early change) was significantly associated with hospital and ICU mortality.

Recently, in a meta-regression analysis from 87 RCTs on septic patients using different SOFA derivatives as primary or secondary endpoints, the authors have shown that $\Delta_{\text {SOFA }}$ (when defined as a fixed day minus initial day SOFA) explained 32\% of treatment effect on mortality, suggesting that $\Delta_{\text {SOFA }}$ is both responsive and consistent in detecting differences of treatment effects on mortality and could replace mortality as a surrogate endpoint in clinical trials [17]. The validity of change of SOFA on day 7 as an early predictor of 28-day mortality was analyzed in a large post-marketing survey among patients with sepsis and disseminated intravascular coagulation, who were propensity-matched to receive either antithrombin III alone or combination therapy with thrombomodulin. Although no difference was found between the two groups, when they were analyzed together, it was found that these changes between day 1 and day 7 provided AUROC 0.81 for 28-day mortality [18]. In a cohort of severe sepsis and septic shock, day 3 $\Delta_{\text {SOFA }}$ displayed AUROC $0.68 \quad(95 \%$ CI $\quad 056-0.79)$ whereas 50\% SOFA decrease was associated with $61.3 \%$ sensitivity and $85.9 \%$ negative predictive value for ICU mortality prediction [19].
Another suggested endpoint based on SOFA score is the mean total SOFA score. This is the sum of the follow-up day SOFA scores divided by the number of days of ICU stay. In an historical cohort of 352 patients with mean length of stay (LOS) of 6.5 days, the mean total SOFA correlated well with mortality (OR 3.06, 95\% CI 2.36 to 3.97) [15]. In a study evaluating levosimendan compared to placebo in patients with septic shock (the LeoPARDS RCT), the primary endpoint was powered to detect an absolute difference in the mean SOFA score (calculated up to a maximum of 28 ICU days) of at least 0.5 between the two arms [20]. The MaxSep RCT, comparing meropenem alone or in combination with moxifloxacin, in patients with severe sepsis, aimed to demonstrate a minimum of 1.1 point difference in mean SOFA scores between the two arms (calculated for a maximum ICU stay of 14 days) [21]. Both studies failed to demonstrate the expected difference, despite adequately large sample sizes (more than 500 patients per study), possibly due to the cutoffs used.

In the light of the existing publications, it is obvious that the suggested cutoff of at least 25\% decrease of SOFA score on day 7 may neither replace mortality as an endpoint of clinical trials nor be considered a surrogate for sepsis resolution. However, there is no doubt that it may be considered as an early marker of improvement of the sepsis process so as to be encountered alongside mortality.

One major limitation of our study is the retrospective analysis of the data. However, due to the fact that all included patients were part of a prospective follow-up protocol during the initial randomized clinical trials, all required data were systematically collected up to day 28 limiting the bias that may come from this approach.

\section{Conclusions}

Overtime changes in Sequential Organ Failure Assessment score $\left(\Delta_{\text {SOFA }}\right)$ offer a more direct, scalar measurement of treatment effect of sepsis compared to 
traditional mortality endpoints. Any less than $25 \% \Delta_{\text {SOFA }}$ on day 7 may identify high mortality-risk patients showing that $\Delta_{\text {SOFA }}$ changes may be incorporated alongside mortality in future clinical trials.

\section{Supplementary information}

Supplementary information accompanies this paper at https://doi.org/10. 1186/s13054-019-2665-5.

\section{Additional file 1: Table S1. Comparative demographics of the two} novel cohorts.

Additional file 2: Table S2. Prognostic performance for 90-day mortality of the $25 \%$ SOFA decrease cutoff on day $7 \Delta_{\text {SOFA }}$ between the derivation and validation cohorts.

\section{Abbreviations}

APACHE: Acute Physiology and Chronic Health Evaluation; ARDS: acute respiratory distress syndrome; AUROC: Area under the receiver operating characteristics curve; CCl: Charlson's comorbidity index; Cl: Confidence

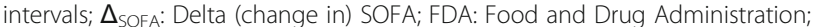
FNIH: Foundation for the National Institutes of Health; GCS: Glasgow Coma Scale; HIV: Human immunodeficiency virus; ICU: Intensive care unit; LOS: Length of stay ; MV: mechanical ventilation; NPV: Negative predictive value; OR: Odds ratio; PPV: Positive predictive value; RCT: Randomized clinical trial; ROC: Receiver operating characteristics; SD: Standard deviation; SIRS: Systemic inflammatory response syndrome; SOFA: Sequential Organ Failure Assessment; VAP: Ventilator-associated pneumonia

\section{Acknowledgements}

Not applicable

\section{Authors' contributions}

EKa and EKy contributed to the collection and analysis of the data, drafted the manuscript, and gave the final approval of the version to be published. $\mathrm{CR}$ and IT contributed to the enrollment of patients and collection of clinical data, participated in the drafting of the manuscript, and gave the final approval of the version to be published. JLV conceptualized the study design, critically revised the manuscript, and gave the final approval of the final version to be published. EJGB conceptualized the study design, contributed to the analysis of the data, participated in the drafting of the manuscript, critically reviewed the manuscript, and gave final approval of the version to be published.

\section{Funding}

The study was funded by unrestricted educational grants provided by the Hellenic Institute for the Study of Sepsis and, by the Horizon 2020 Marie Skłodowska-Curie Grant European Sepsis Academy (grant 676129).

\section{Availability of data and materials}

The datasets used and/or analyzed during the current study are available from the corresponding author upon reasonable request.

\section{Ethics approval and consent to participate}

Written informed consent was provided from all participants or their legal representatives. The derivation cohort resulted from a study approved by the Central Ethics Committee (EED), Athens, Greece (approval 42797/20-06-2007), and the National Organization for Medicines (EOF), Athens, Greece (approval 76305/15-02-2007), and was conducted according to the Declaration of Helsinki. EudraCT number 2006-004886-33. The validation cohort came from a study approved by the National Organization for Medicines of Greece (approval 14653/14-6-2004) that was conducted according to the Declaration of Helsinki.

\section{Consent for publication}

Not applicable

\section{Competing interests}

E Karakike is funded by the Horizon 2020 Marie Skłodowska-Curie Grant European Sepsis Academy (grant 676129 paid to the University of Athens). EJ Giamarellos-Bourboulis has received honoraria (paid to the University of Athens) from AbbVie USA, Abbott $\mathrm{CH}$, Biotest Germany, Brahms GmbH, InflaRx GmbH, the Medicines Company, MSD Greece, and XBiotech Inc. He has received independent educational grants from AbbVie, Abbott, Astellas Pharma, AxisShield, bioMérieux Inc., InflaRx GmbH, the Medicines Company, and XBiotech Inc. He has received funding from the FrameWork 7 program HemoSpec and from the Horizon 2020 Marie-Curie project European Sepsis Academy (granted to the National and Kapodistrian University of Athens), outside the submitted work.

The other authors declare that they have no competing interests.

\section{Author details}

${ }^{1} 4$ th Department of Internal Medicine, Attikon University Hospital, National and Kapodistrian University of Athens, 1 Rimini Street, 12462 Athens, Greece. ${ }^{2}$ 2nd Department of Critical Care Medicine, National and Kapodistrian University of Athens, 12462 Athens, Greece. ${ }^{3} 1$ st Department of Critical Care Medicine, National and Kapodistrian University of Athens, 10676 Athens, Greece. ${ }^{4}$ Department of Intensive Care, Erasme Hospital, Université libre de Bruxelles, 1070 Brussels, Belgium.

Received: 17 July 2019 Accepted: 7 November 2019 Published online: 29 November 2019

\section{References}

1. Ranieri VM, Thompson BT, Barie PS, Dhainaut JF, Douglas IS, Finfer S, et al. Drotrecogin alfa (activated) in adults with septic shock. N Engl J Med. 2012; 366:2055-64.

2. Payen DM, Guilhot J, Launey Y, Lukaszewicz AC, Kaaki M, Veber B, et al. Early use of polymyxin $B$ hemoperfusion in patients with septic shock due to peritonitis: a multicenter randomized control trial. Intensive Care Med. 2015; 41:975-84.

3. Sprung $\mathrm{CL}$, Annane $\mathrm{D}$, Keh $\mathrm{D}$, Moreno $\mathrm{R}$, Singer $\mathrm{M}$, Freivogel $\mathrm{K}$, et al Hydrocortisone therapy for patients with septic shock. N Engl J Med. 2008; 358:111-24.

4. Mouncey PR, Osborn TM, Power GS, Harrison DA, Sadique MZ, Grieve RD, et al. Trial of early, goal-directed resuscitation for septic shock. N Engl J Med. 2015;372:1301-11.

5. Rhodes A, Evans LE, Alhazzani W, Levy MM, Antonelli M, Ferrer R, et al. Surviving Sepsis Campaign: international guidelines for management of sepsis and septic shock: 2016. Intensive Care Med. 2017;43:304-77.

6. U. S. Food Drug Administration F. Guidance for industry. Acute bacterial skin and skin structure infections: developing drugs for treatment. Silver Spring: Food and Drug Administration; 2013. http://www.fda.gov/downloads/ Drugs/./Guidances/ucm071185.pdf. Accessed 15 Nov 2017

7. U. S. Food Drug Administration F. Guidance for industry: communityacquired bacterial pneumonia: developing drugs for treatment, Center for Drug Evaluation and Research. Silver Spring: Food and Drug Administration, US Department of Health and Human Services; 2014 Accessed 15 Nov 2017

8. Foundation for the National Institutes of Health Biomarkers Consortium HABPNABP Working Group, 2013. Interim considerations for clinical trial design for the study of hospital-acquired bacterial pneumonia and ventilator-associated bacterial pneumonia. Submission to Docket FDA-2013N-0556. https://http://www.pharmamedtechbi.com/ /media/Supporting Documents/The Pink Sheet/75/36/fnihcommentsantibacterial.pdf. Accessed 15 Nov 2017.

9. Talbot $\mathrm{GH}$, Powers $\mathrm{JH}$, Hoffmann SC, Biomarkers Consortium of the Foundation for the National Institutes of Health C-A, Teams H-VP. Developing outcomes assessments as endpoints for registrational clinical trials of antibacterial drugs: 2015 update from the biomarkers consortium of the foundation for the national institutes of health. Clin Infect Dis. 2016;62:603-7.

10. Prescott HC, Osterholzer JJ, Langa KM, Angus DC, Iwashyna TJ. Late mortality after sepsis: propensity matched control study. BMJ. 2016; 353:i2375.

11. Bone RC, Balk RA, Cerra FB, Dellinger RP, Fein AM, Knaus WA, et al. Definitions for sepsis and organ failure and guidelines for the use of innovative therapies in sepsis. The ACCP/SCCM Consensus Conference 
Committee American College of Chest Physicians/Society of Critical Care Medicine. Chest. 1992;101:1644-55.

12. Giamarellos-Bourboulis EJ, Mylona V, Antonopoulou A, Tsangaris I, Koutelidakis I, Marioli A, et al. Effect of clarithromycin in patients with suspected Gram-negative sepsis: results of a randomized controlled trial. J Antimicrob Chemother. 2014;69:1111-8.

13. Giamarellos-Bourboulis EJ, Pechere JC, Routsi C, Plachouras D, Kollias S, Raftogiannis $\mathrm{M}$, et al. Effect of clarithromycin in patients with sepsis and ventilator-associated pneumonia. Clin Infect Dis. 2008;46:1157-64.

14. Minne L, Abu-Hanna A, de Jonge E. Evaluation of SOFA-based models for predicting mortality in the ICU: a systematic review. Crit Care. 2008;12:R161.

15. Ferreira FL, Bota DP, Bross A, Melot C, Vincent JL. Serial evaluation of the SOFA score to predict outcome in critically ill patients. JAMA. 2001;286: 1754-8.

16. Soo A, Zuege DJ, Fick GH, Niven DJ, Berthiaume LR, Stelfox HT, et al. Describing organ dysfunction in the intensive care unit: a cohort study of 20,000 patients. Crit Care. 2019;23:186.

17. de Grooth HJ, Geenen IL, Girbes AR, Vincent JL, Parienti JJ, Oudemans-van Straaten HM. SOFA and mortality endpoints in randomized controlled trials: a systematic review and meta-regression analysis. Crit Care. 2017;21:38.

18. Iba T, Arakawa M, Mochizuki K, Nishida O, Wada H, Levy JH. Usefulness of measuring changes in SOFA score for the prediction of 28-day mortality in patients with sepsis-associated disseminated intravascular coagulation. Clin Appl Thromb Hemost. 2019:25:1076029618824044.

19. de Azevedo JR, Torres OJ, Beraldi RA, Ribas CA, Malafaia O. Prognostic evaluation of severe sepsis and septic shock: procalcitonin clearance vs delta sequential organ failure assessment. J Crit Care. 2015;30(219):e9-12.

20. Gordon AC, Perkins GD, Singer M, McAuley DF, Orme RM, Santhakumaran S, et al. Levosimendan for the prevention of acute organ dysfunction in sepsis. N Engl J Med. 2016;375:1638-48.

21. Brunkhorst FM, Oppert M, Marx G, Bloos F, Ludewig K, Putensen C, et al. Effect of empirical treatment with moxifloxacin and meropenem vs meropenem on sepsis-related organ dysfunction in patients with severe sepsis: a randomized trial. JAMA. 2012;307:2390-9.

\section{Publisher's Note}

Springer Nature remains neutral with regard to jurisdictional claims in published maps and institutional affiliations.

Ready to submit your research? Choose BMC and benefit from:

- fast, convenient online submission

- thorough peer review by experienced researchers in your field

- rapid publication on acceptance

- support for research data, including large and complex data types

- gold Open Access which fosters wider collaboration and increased citations

- maximum visibility for your research: over $100 \mathrm{M}$ website views per year

At $\mathrm{BMC}$, research is always in progress.

Learn more biomedcentral.com/submissions 